\title{
What are the requirements for a manuscript to be published in a journal specialized in a multidisciplinary health issue?
}

\author{
JJ Wyndaele, Editor \\ Antwerp University Hospital, Antwerp, Belgium \\ E-mail: spinalcord@uza.be
}

Dear Spinal Cord reader,

This July issue reflects fully the complexity and multidisciplinary nature of the care of patients with a spinal cord lesion. It is, of course, unthinkable that various specialists in each 'small' field would independently try to help a patient. The management has to be comprehensive, guided and performed by the core rehabilitation group, which is supported by several specialists. Each spinal cord physician, nurse, occupational therapist, physiotherapist and psychologist must be aware of as many of the dysfunctions that can occur after a spinal cord lesion as possible; therefore, all specialized team members are expected to have some knowledge about the complete care and about the complex dysfunctions that will or can occur. They, therefore, require a lot of knowledge, and this can be acquired by direct education, from experience, from group discussions and from observation. Knowledge can also be gained through reading.

Spinal Cord, as the international voice of the spinal cord, offers a wealth of knowledge to a specialized readership each month. It is essential that readers find what they are looking for: both specialized and general information, which will help them to improve their work and provide them with a better understanding of patient issues. Much care is, therefore, taken when putting the Spinal Cord issues together and to ensure data of high value is published.

It is easy to understand why authors who submitted their work to our Journal are disappointed when it is not accepted for publication. Criteria for acceptance include relevance to the field, quality of the study design, scientific and clinical interest and excellence of writing. Main reasons for rejection are poor experimental design or inadequate investigation, failure to conform to the Journal guidelines, very poor language, grammar and syntax, inaccurate statement of the aim, poor description of the methods used or over interpretation of the results. Correct statistics have also become crucial. Confusing or inadequate presentation in tables and figures, or there being too few or too many can cause problems. Reviewers are specialists and they know the related literature well: incomplete, outdated or inaccurate literature reviews will give a very poor impression.

This seems to be a very long list of requirements but they are within reach as proven by the high standards of the work submitted to Spinal Cord. Many working in the field of spinal cord medicine will find the data published in this issue of direct value to their daily work. This month's review discusses risk factors for coronary heart disease in individuals with spinal cord injury, a major life risk factor. Metabolic syndrome, altered innate immunity, comparison of rehabilitation between patients with traumatic and non-traumatic lesions will interest many of you. One manuscript has the challenging title: 'who wants to walk?'. The next international basic spinal cord injury data set, this time on urodynamics, continues this extremely interesting line of work.

Have a look at the list of articles in this issue and you will find additional very good research. Enjoy reading

Spinal Cord (2008) 46, 465; doi:10.1038/sc.2008.80 